TRABAJOS ORIGINALES

Rev Obstet Ginecol Venez. 2021; 81 (2): 137-147

https://doi.org/10.51288/00810207

\title{
Periodo intergenésico como factor de riesgo para el desarrollo de preeclampsia
}

\author{
Carli Solé Aquino, ${ }^{1}$ Nancy Estévez, ${ }^{1}$ (D) Harold Guevara Rivas. ${ }^{2}$
}

RESUMEN

Objetivo: Determinar el periodo intergenésico como factor de riesgo para el desarrollo de preeclampsia en gestantes atendidas en una Maternidad de Alto Riesgo entre enero y junio de 2018.

Métodos: Se realizó una investigación retrospectiva, transversal, analítica de 160 casos y 161 controles a partir de historias clínicas.

Resultados: Las medianas de las edades fueron 28,00 \pm 9,00 años para los casos y 38,00 $\pm 2,00$ años para los controles ( $p=0,000)$. El antecedente personal más frecuente fue la hipertensión arterial (6,3\%, 10 mujeres). Los antecedentes familiares más relevantes fueron hipertensión arterial $(13,8 \%, 22)$ y diabetes $(13,1 \%, 21)$. El antecedente obstétrico más frecuente fue la cesárea anterior $(67,5 \%, 108$ gestantes $)(Z=6,16 ; p=0,00)$. La preeclampsia con criterio de gravedad predominó estadísticamente $(93,8 \%, 150$ pacientes) $(Z=15,54$ y $p=0,00)$. La eclampsia fue la complicación más frecuentes con 5,6\% (9 gestantes). El 91,3\% (146 pacientes) no presentó ninguna complicación. La mediana del periodo intergenésico fue 60,00 $\pm 63,75$ meses para los casos y 38,00 $\pm 33,00$ para los controles $(p=0,11)$. Fue corto en 41 casos $(25,6 \%)$ y 26 controles (16,1\%); adecuado en 33 (20,6\%) casos y 84 (52,2\%) controles y prolongado en 86 casos $(53,8 \%)$ y 51 controles $(31,7 \%)(p=0,00)$. Tener un periodo intergenésico inadecuado (corto o prolongado) constituyó un factor riesgo 4,26 veces mayor de tener la PE en comparación (OR=4,26; IC 95\%: 2,59-6,98).

Conclusiones: Tener un periodo intergenésico inadecuado eleva cuatro veces el riesgo de desarrollar preeclampsia.

Palabras clave: Hipertensión, Preeclampsia, Periodo intergenésico.

\section{SUMMARY}

Objective: To determine the intergenic period as a risk factor for development preeclampsia in pregnant women attended in a High Risk Maternity in the period January-June 2018.

Methods: A retrospective, transversal, analytical investigation was carried out of 160 cases and 161 controls, using a data collection form based on medical records.

Results: The median ages were $28.00 \pm 9.00$ years for cases and $38.00 \pm 2.00$ years for controls $(p=0.000)$. The most frequent personal history was arterial hypertension (6.3\%, 10 women). The most relevant family history was arterial hypertension $(13.8 \%, 22)$ and diabetes $(13.1 \%, 21)$. The most frequent obstetric antecedent was previous caesarean section $(67.5 \%, 108$ pregnant women) $(Z=6.16 ; p=0.00)$. Preeclampsia with severity criteria predominated statistically $(93.8 \%, 150$ patients $)$ $(Z=15.54$ and $p=0.00)$. Eclampsia was the most frequent complication with $5.6 \%$ (9 pregnant women). The $91.3 \%(146$ patients) did not present any complications. The median intergenic period was $60.00 \pm 63.75$ months for cases and $38.00 \pm$ 33.00 for controls $(p=0.11)$. It was short in 41 cases $(25.6 \%)$ and 26 controls $(16.1 \%)$; adequate in $33(20.6 \%)$ cases and $84(52.2 \%)$ controls and prolonged in 86 cases $(53.8 \%)$ and 51 controls $(31.7 \%)(p=0.00)$. Having an inadequate intergenic period (short or prolonged) was a risk factor 4.26 times higher for having PE compared (OR=4.26; 95\% CI: 2.59- 6.98). Conclusions: Having an inadequate intergenic period increases the risk of developing preeclampsia by four times.

Keywords: Hypertension, preeclampsia, intergenic period.

\section{INTRODUCCIÓN}

${ }^{1}$ Especialistas en Ginecología y Obstetricia. Ciudad Hospitalaria Dr. Enrique Tejera. Maternidad de Alto Riesgo. Valencia, estado Carabobo. Venezuela. ${ }^{2}$ Doctor en Ciencias Médicas. Profesor Titular. Departamento de Salud Pública, sede Carabobo. Universidad de Carabobo. Valencia, Venezuela.
La prevención y el control de la hipertensión inducida por el embarazo (HIE) es considerada por la Organización Mundial de la Salud (OMS) como 
una prioridad de salud global. Es una enfermedad que se presenta únicamente en la gestación humana, influyendo directamente en las condiciones de salud del binomio madre-hijo, no solo a corto plazo, es decir, generándose mortalidad materna y perinatal, sino a largo plazo, con el incremento del riesgo cardiovascular de las madres y en la presentación de la mortalidad neonatal (1).

Cada día mueren unas 800 mujeres por causas prevenibles relacionadas con el embarazo y el parto. La mayoría de esas complicaciones aparecen durante la gestación; otras pueden estar presentes antes del embarazo, pero se agravan con el mismo, las principales causas de mortalidad materna ocasionan el $80 \%$ de estas muertes, entre ellas la preeclampsia (PE) (2).

La preeclampsia/eclampsia es una de las principales causas de morbimortalidad materna y perinatal del mundo. Su incidencia es de $7 \%$ al $10 \%$ y actualmente se calcula que por su causa se producen 200000 muertes maternas por año (3). Respecto al periodo intergenésico, algunos estudios señalan que es un factor de riesgo para la madre en el desarrollo de PE (4-7).

En Estados Unidos, representa al menos $15 \%$ de las muertes relacionadas con embarazo (8). En países en vías de desarrollo, su incidencia y complicaciones son mucho más elevadas. En Colombia, donde la mortalidad materna asociada a $\mathrm{PE}$ es 10 veces mayor que en Estados Unidos, su incidencia está entre $13 \%$ y $18 \%$ (9); en México es la complicación más frecuente del embarazo, la incidencia es 47,3 por cada 1000 nacimientos y es la primera causa de ingreso de embarazadas a las unidades de terapia intensiva (10).

En 2005, según la Organización Panamericana de la Salud, Venezuela tuvo una tasa de mortalidad materna de 59,9 fallecidas por cada 100000 nacidos vivos registrados y el $28,6 \%$ fue por trastornos hipertensivos del embarazo, correspondiendo a la primera causa de muerte materna a nivel nacional; de esto no escapa Carabobo, donde los trastornos hipertensivos del embarazo son la primera causa de morbimortalidad materna y perinatal (11).

Según el Reporte del Programa Americano de Educación de Hipertensión en Embarazadas (12), del año 2000, la PE se define como una enfermedad compleja, en la que un conjunto de factores medio ambientales (nutricionales, infecciosos, inmunológicos) en asociación con una susceptibilidad genética, determinan su presentación y curso. Al respecto López y cols. (13), identificaron como factores de riesgo asociados a PE el sobrepeso/obesidad, cesárea o PE en embarazo previo, control prenatal irregular y periodo intergenésico corto o largo. Por otro lado, Mosquera y cols. (14), identificaron los principales factores de riesgo asociados a PE en gestantes de un hospital, obteniendo que no se demostró significancia estadística en las variables estudiadas: edad, área de residencia, escolaridad, controles prenatales, paridad, gestaciones, abortos, cesáreas, periodo intergenésico, malnutrición, clasificación del riesgo obstétrico, antecedentes patológicos personales y familiares.

Por su parte, Centeno y cols. (15) determinaron que existe mayor riesgo de $\mathrm{PE}$ en mujeres con periodo intergenésico prolongado (mayor a 48 meses). También Sedano y cols. (16) demostraron asociación significativa entre el periodo intergenésico prolongado y preeclampsia, además que eleva 4 veces el riesgo de padecer esta enfermedad.

La etiología de la PE se desconoce, no obstante hay cuatro hipótesis en la etiopatogenia de la enfermedad hipertensiva del embarazo: 1) mala adaptación inmunológica 2) isquemia placentaria, 3) estrés oxidativo y 4) susceptibilidad genética $(4,17$ - 21).

En cuanto a la mala adaptación inmunológica se postula que el riesgo de PE disminuye después del 
primer embarazo (excepto si el periodo intergenésico es mayor a 10 años) así como un efecto protector de la multiparidad (exposición frecuente a semen), uso de dispositivos intrauterinos (DIU) o anticonceptivos orales, transfusiones sanguíneas y abortos frecuentes $(4,17$ - 19).

La isquemia placentaria se debe a la falta de relajación de las arterias espirales. La exportación incrementada de membranas de microvellosidades del sincitiotrofoblasto (STBM) en mujeres preeclámpticas daña al endotelio e inhiben su proliferación. Esta, además, causa un estrés oxidativo importante sobre el endotelio vascular (4, 17 - 19).

El sustrato genético y las alteraciones inmunológicas participan en la formación de un síndrome inflamatorio y metabólico caracterizado por lesión endotelial como centro fisiopatológico, que se evidencia clínicamente en la forma de hipertensión, proteinuria, alteraciones de la coagulación e hipoperfusión tisular generalizada, que puede conducir finalmente a la disfunción orgánica múltiple y a la muerte materna y/o perinatal (13).

El periodo intergenésico tiene que ver con la alteración de la tolerancia inmunológica, que normalmente se produce durante el embarazo, el cual influye en el proceso normal de vasculogénesis, formación del tejido placentario y en la producción adecuada de factores que regulan los cambios fisiológicos maternos; las alteraciones inmunológicas que afectan a la formación placentaria y culminan en la disfunción endotelial son las que marcan el paso final para una serie de fenómenos que desencadenan en la PE (3).

El Colegio Americano de Obstetras y Ginecólogos, en 2018, define PE como la existencia de presión arterial sistólica $\geq 140 \mathrm{mmHg}$ y/o diastólica $\geq 90 \mathrm{mmHg}$, en al menos dos tomas con 4 horas de separación, asociada a proteinuria $\geq 300 \mathrm{mg}$ en 24 horas (o un índice de proteinuria creatinina -IPC- $\geq 0,3$ en una muestra aislada), en pacientes embarazadas, con edad gestacional de al menos 20 semanas, y cifras tensionales normales previo al embarazo (21). Ante signos de gravedad, como presencia de síndrome de HELLP (hemólisis, enzimas hepáticas elevadas, trombocitopenia), falla renal aguda, edema pulmonar o alteración visual o cerebral, se puede prescindir de la determinación de proteinuria para el diagnóstico. En pacientes con cifras tensionales en rango de crisis, ambas tomas pueden ser medidas con un intervalo de minutos para realizar el diagnóstico (Cuadro 1) (21).

Hay algunos factores de riesgo para PE o su recurrencia: historia personal de hipertensión arterial, diabetes gestacional o enfermedades autoinmunes; historia personal o familiar de PE, nuliparidad, embarazo múltiple, sobrepeso u obesidad previa al embarazo, malformaciones fetales, cambio de pareja. Asimismo, son factores protectores: índice de masa corporal previo al embarazo $<19 \mathrm{~kg} / \mathrm{m}^{2}$ y el tabaquismo durante el embarazo (22).

Hay otros factores que se han explorado, para los cuales no existe consenso sobre su relación con la PE. Entre ellos están: menor edad gestacional en el primer parto, periodo intergenésico, sexo fetal, infecciones durante la gestación, estrés, la violencia intrafamiliar o que el embarazo sea producto de reproducción asistida (22).

La PE es causa de morbimortalidad materna, la falla endotelial puede ocurrir en cualquier nivel del organismo, pudiendo ocasionar hemorragia posparto, desprendimiento prematuro de placenta, coagulopatía, insuficiencia renal, encefalopatía hipertensiva, hemorragia intracerebral, síndrome HELLP y rotura de hematoma hepático $(20,23,24)$.

Las complicaciones perinatales pueden ocurrir durante el embarazo, el parto o después de nacer. La prematuridad, la restricción de crecimiento intrauterino, el bajo peso al nacer, el sufrimiento fetal y la muerte fetal representan alrededor del $10 \%$ a $40 \%$ (25). 
Cuadro 1. Criterios diagnósticos de preeclampsia con y sin criterios de gravedad (21)

\begin{tabular}{|c|c|c|}
\hline Marcador clínico & Preeclampsia & Preeclampsia con criterios de gravedad \\
\hline Presión arterial & $\begin{array}{c}\mathrm{PAS} \geq 140 \mathrm{y}<160 \mathrm{y} / \mathrm{o} \\
\mathrm{PAD} \geq 90 \mathrm{y}<110\end{array}$ & $\mathrm{PAS} \geq 160 \mathrm{y} / \mathrm{o}$ PAD $\geq 110$ \\
\hline Proteinuria & $>300 \mathrm{mg} /$ día o IPC 0,3 & No aplica \\
\hline Diuresis & > 500 ml/día & $\leq 500 \mathrm{ml} /$ día \\
\hline Compromiso sistémico & No & $\begin{array}{c}\text { Edema pulmonar, insuficiencia renal } \\
\text { aguda, hematoma subcapsular hepá- } \\
\text { tico, dolor en epigastrio, náuseas o } \\
\text { vómitos. }\end{array}$ \\
\hline Compromiso neurológico & No & $\begin{array}{l}\text { Convulsiones, cefalea, fotopsia, cegue- } \\
\text { ra visión borrosa, alteración estado } \\
\text { mental. }\end{array}$ \\
\hline Compromiso de laboratorios & No & $\begin{array}{c}\text { Plaquetas }<100000 \text { GOT }>70 \\
\text { LDH }>2 \text { veces el VR }\end{array}$ \\
\hline Compromiso fetal & No & Restricción del crecimiento fetal. \\
\hline
\end{tabular}

PAS: presión arterial sistólica; PAD: presión arterial diastólica; IPC: índice de proteinuria/creatinina en muestra de orina aislada; VR: valor de referencia laboratorio local.

Respecto al periodo intergenésico, es el lapso entre fechas de nacimientos consecutivos $(3,5)$, aunque otro autor lo define como el tiempo entre fechas de dos nacimientos vivos, sucesivos o consecutivos menos la edad gestacional del neonato más reciente $(6,8,26)$. La longitud de los intervalos se ve afectada por factores que influyen en el espaciamiento de los nacimientos, entre ellos están: la edad de la madre al momento del nacimiento del hijo, el nivel socioeconómico, la educación materna, el uso de algún método anticonceptivo y la procedencia (26). El tener nacimientos demasiado próximos no permite que el cuerpo de la madre recupere las reservas nutricionales y el nivel energético, provocando así embarazos riesgosos, complicados en la mayoría de los casos por situaciones como: enfermedad hipertensiva del embarazo, parto obstruido o hemorragia posparto (26).

El periodo intergenésico se clasifica como corto cuando es menor o igual a 24 meses, entre 25 y 48 meses se considera adecuado y mayor o igual a 49 meses como prolongado (5), aunque otros estudios lo clasifican como corto si es menor de 24 meses, entre 24 y 48 meses como lo normal y mayor de 48 meses como el prolongado (3).

La preeclampsia es una de las complicaciones más frecuentes que afecta a las gestantes en Venezuela, de allí surge la necesidad de conocer los diferentes factores asociados a esta enfermedad, siendo prioridad y objeto del presente estudio el periodo intergenésico como factor de riesgo importante para su desencadenamiento. El efecto del periodo intergenésico en complicaciones maternas como la PE, se ha estudiado poco en países en vías de desarrollo, por tal razón se pretendió identificar si este factor es un riesgo significativo para las gestantes; para así plantear medidas preventivas y de promoción de la salud pertinentes y oportunas, siendo esto importante para identificar a aquellas gestantes que estarían más expuestas a desarrollar esta patología y, por lo tanto, tener en cuenta el riesgo que ellas puedan correr. 
En el Estado Carabobo se han realizado estudios sobre este problema, pero tomando en cuenta otros factores asociados a la PE, siendo el periodo intergenésico poco explorado. Romero (27) evaluó la prevalencia en adolescentes gestantes con PE severa en la Maternidad sede de este estudio. Otro estudio en la misma maternidad es el de León (28), quien estableció la incidencia de preeclampsia en pacientes con antecedentes de trastorno hipertensivo en embarazos previos.

Este trabajo se planteó analizar si el periodo intergenésico es un factor de riesgo para el desarrollo de preeclampsia, es decir, categorizar el periodo intergenésico y estudiar la asociación entre el periodo intergenésico y la preeclampsia. Además, identificar las características maternas, antecedentes obstétricos, antecedentes de riesgo de preeclampsia y características del embarazo actual.

\section{MÉTODOS}

Se realizó un estudio retrospectivo, transversal, analítico, tipo casos y controles.

La población la integraron las gestantes que presentaban preeclampsia durante su embarazo y fueron atendidas en la Maternidad de Alto Riesgo Comandante Supremo Hugo Chávez, en el periodo enero-junio de 2018. La muestra fue no probabilística, intencional, con gestantes divididas en dos grupos: 160 casos de gestantes con diagnóstico de preeclampsia y 161 controles de gestantes sin diagnóstico de preeclampsia, siendo la unidad de muestreo la historia clínica de la paciente. Los criterios de inclusión fueron, para los casos: gestantes segunda gestas o más, con edad gestacional $>20$ semanas, con diagnóstico de preeclampsia según criterios establecidos por la institución. Para los controles: gestantes segunda gestas o más, con edad gestacional $>20$ semanas, sin diagnóstico de preeclampsia. Los criterios de exclusión fueron antecedentes patológicos (insuficiencia renal o hepática) y registros incompletos.

El instrumento de recolección de datos constó de cuatro partes. La primera permitió recolectar datos generales de la paciente, como edad, nivel de educación, estado civil. En la segunda parte se tomaron los antecedentes de la paciente tales como los personales, familiares y obstétricos. En la tercera parte se encontraban los datos del embarazo y parto actual. En esta investigación se asumió como criterio para medir el periodo intergenésico, el tiempo que existe entre fechas de nacimientos consecutivos. En el cuarto punto, los datos relacionados con el diagnóstico de preeclampsia, según los criterios establecidos en la institución.

Los datos se procesaron con el paquete estadístico PAST 3.17. Todas las variables cuantitativas presentaban distribución libre (no se adaptaron a la distribución normal), previa confirmación con la prueba de Kolmogorov-Smirnov, por lo cual se describieron con la mediana y el rango intercuartil. Se realizaron comparaciones de medianas entre muestras independientes con la prueba no paramétrica de MannWhitney (MW) y comparaciones de proporciones con la prueba Z. Se buscó asociación entre las variables cualitativas con el chi cuadrado $\left(\chi^{2}\right)$ con corrección de Yates en tablas 2 × 2 y sin corrección cuando no se requería. Se estimó el riesgo relativo con el odds ratio (OR) y sus respectivos intervalos de confianza para un $95 \%$. Se asumió para todas las pruebas un nivel de significancia de $\mathrm{p}<0,05$.

\section{RESULTADOS}

Se estudió una muestra de 321 gestantes, divididas en dos grupos. Los casos fueron 160 pacientes y los controles, 161 pacientes. En la tabla 1 se observa que, entre los casos, el grupo etario más frecuente fue el de 19 a 29 años (52,5\%, 84 mujeres) y en los controles 
fue el de 30 a 42 años (96,9 \%, 156 embarazadas). Las medianas de las edades fueron $28,00 \pm 9,00$ años para los casos y $38,00 \pm 2,00$ años para los controles $(\mathrm{p}=0,000)$.

La mayor proporción de pacientes con preeclampsia cursaron bachillerato completo $(46,9 \%, 75)$, $72,5 \%$ (116 gestantes) eran solteras, con predominio estadísticamente significativo $(Z=7,94 ; \mathrm{p}=0,00)$, y $44,4 \%$ (71 embarazadas) procedían del municipio Valencia (Tabla 2). El antecedente personal más frecuente fue la hipertensión arterial $(6,3 \%, 10$ mujeres). Los antecedentes familiares más relevantes fueron hipertensión arterial $(13,8 \%, 22)$ y diabetes $(13,1 \%, 21)$. El antecedente obstétrico más frecuente fue la cesárea anterior $(67,5 \%, 108$ gestantes $)(Z=6,16$; $\mathrm{p}=0,00)$. El 63,4 \% (102 pacientes) cambiaron de pareja sexual $(\mathrm{Z}=4,81 ; \mathrm{p}=0,00)$ y $3,1 \%$ (5 gestantes) eran fumadoras (Tabla 3 ).

La preeclampsia con criterio de gravedad predominó estadísticamente $(93,8 \%, 150$ pacientes) $(Z=15,54$ y $\mathrm{p}=0,00)$. El síntoma más frecuente asociado a la preeclampsia fue la cefalea $(66,3 \%, 106$ mujeres; $\mathrm{Z}=5,70 ; \mathrm{p}=0,00)$. La eclampsia fue la complicación más frecuentes con $5,6 \%$, es decir, 9 gestantes afectadas. El 91,3 \% (146 pacientes) no presentó ninguna complicación (Tabla 4).

Tabla 1. Distribución comparativa de las gestantes según la edad

\begin{tabular}{lcccc}
\hline $\begin{array}{l}\text { Grupo etario } \\
\text { (años) }\end{array}$ & \multicolumn{2}{c}{$\begin{array}{c}\text { Preeclampsia } \\
(\mathrm{n}=160)\end{array}$} & \multicolumn{2}{c}{$\begin{array}{c}\text { Sin preeclampsia } \\
(\mathrm{n}=161)\end{array}$} \\
\cline { 2 - 5 } & $\mathrm{n}$ & $\%$ & $\mathrm{n}$ & $\%$ \\
\hline 15 a 18 & 10 & 6,3 & 0 & 0,0 \\
19 a 29 & 84 & 52,5 & 5 & 3,1 \\
30 a 42 & 66 & 41,3 & 156 & 96,9 \\
\hline$(\mathrm{p}=0,000)$. & & & &
\end{tabular}

Tabla 2. Distribución de los casos según nivel de educación, estado civil y procedencia

\begin{tabular}{lcc}
\hline Característica & $\mathrm{n}$ & $\%$ \\
\hline Nivel de educación & & \\
Primaria incompleta & 1 & 0,6 \\
Primaria completa & 38 & 23,8 \\
Bachillerato incompleto & 5 & 3,1 \\
Bachillerato completo & 75 & 46,9 \\
TSU & 8 & 5,0 \\
Universitario & 33 & 20,6 \\
Estado civil & & \\
Soltera & 116 & $72,5 *$ \\
Unión libre & 25 & 15,6 \\
Casada & 18 & 11,3 \\
Divorciada & 1 & 0,6 \\
Procedencia por Municipio & & \\
Valencia & 71 & 44,4 \\
Libertador & 20 & 12,5 \\
Guacara & 15 & 9,4 \\
Los Guayos & 11 & 6,9 \\
Naguanagua & 9 & 5,6 \\
Carlos Arvelo & 7 & 4,4 \\
San Joaquín & 6 & 3,8 \\
San Diego & 5 & 3,1 \\
Otro & 16 & 10 \\
\hline
\end{tabular}

$* \mathrm{Z}=7,94 ; \mathrm{p}=0,00$

Las medianas de las presiones arteriales fueron significativamente mayores entre las mujeres con preeclampsia en comparación con los controles (Tabla $5 ; \mathrm{p}<0,05)$.

La mediana del periodo intergenésico fue de 60,00 $\pm 63,75$ meses para los casos y 38,00 $\pm 33,00$ para los controles $(U=11554,50 ; p=0,11)$. En la tabla 6 se presenta la distribución comparativa del periodo intergenésico. El mismo fue corto en 41 casos 
Tabla 3. Distribución de las pacientes con preeclampsia según antecedentes personales, familiares, obstétricos, de tabaquismo y si cambió la pareja sexual

\begin{tabular}{lcc}
\hline Datos clínicos & $\mathrm{n}$ & $\%$ \\
\hline Antecedentes personales & 146 & 91,3 \\
Ninguno & 10 & 6,3 \\
Hipertensión arterial & 3 & 1,9 \\
Asma & 1 & 0,6 \\
Neoplasias & & \\
Antecedentes familiares & 104 & 65,0 \\
Ninguno & 22 & 13,8 \\
Hipertensión arterial & 21 & 13,1 \\
Diabetes & 6 & 3,8 \\
Cardiopatía & 3 & 1,9 \\
Neoplasias & 3 & 1,9 \\
Insuficiencia renal & 1 & 0,6 \\
TBC & & \\
Antecedentes obstétricos & 108 & $67,5^{*}$ \\
Cesárea anterior & 21 & 13,1 \\
Ninguno & 19 & 11,9 \\
Preeclampsia & 8 & 5,0 \\
Eclampsia & 4 & 2,5 \\
Ruptura prematura de membranas & 102 & $63,4^{* *}$ \\
Cambio de pareja sexual & 5 & 3,1 \\
Tabaquismo & & \\
\hline
\end{tabular}

$* \mathrm{Z}=6,16 ; \mathrm{P}=0,00$

$* * \mathrm{Z}=4,81 ; \mathrm{P}=0,00$

$(25,6 \%)$ y en 26 controles (16,1\%); adecuado en 33 $(20,6 \%)$ casos y $84(52,2 \%)$ controles y prolongado en 86 casos $(53,8 \%)$ y 51 controles $(31,7 \%)$. Se evidenció asociación estadísticamente significativa entre el periodo intergenésico y la presencia de preeclampsia $\left(\chi^{2}\right.$ de Pearson $\left.=34,53 ; p=0,00\right)$.

Al dicotomizar el periodo intergenésico en adecuado e inadecuado (corto o prolongado), se corroboró la asociación estadísticamente significativa entre el periodo intergenésico inadecuado y la presencia de
Tabla 4. Distribución de los casos según el grado de la preeclampsia, síntomas y complicaciones

\begin{tabular}{lcc}
\hline Grado de preeclampsia & $\mathrm{n}$ & $\%$ \\
\hline Sin criterio de gravedad & 10 & 6,3 \\
$\quad$ Con criterio de gravedad & 150 & $93,8^{*}$ \\
Síntomas de preeclampsia & & \\
$\quad$ Cefalea & 106 & $66,3^{* *}$ \\
Dolor abdominal & 31 & 19,4 \\
Escotomas & 10 & 6,3 \\
Ninguno & 7 & 4,4 \\
Tinnitus & 6 & 3,8 \\
Complicaciones & & \\
Ninguna & 146 & $91,3 * * *$ \\
Eclampsia & 9 & 5,6 \\
HELLP & 4 & 2,5 \\
Síncope & 1 & 0,6 \\
\hline *Z $=15,54 ; \mathrm{P}=0,00$ & & \\
$* * \mathrm{Z}=5,70 ; \mathrm{P}=0,00$ & & \\
& &
\end{tabular}

Tabla 5. Comparación de medianas de presiones arteriales y periodo intergenésico entre las gestantes con y sin preeclampsia

\begin{tabular}{lccc}
\hline $\begin{array}{l}\text { Variable } \\
(\mathrm{Md} \pm \mathrm{RIC})^{*}\end{array}$ & $\begin{array}{c}\text { Preeclampsia } \\
(\mathrm{n}=160)\end{array}$ & $\begin{array}{c}\text { Sin } \\
\text { preeclampsia } \\
(\mathrm{n}=161)\end{array}$ & $\mathrm{U} ; * * / \mathrm{p}$ \\
\hline $\begin{array}{l}\text { Presión arterial } \\
\text { sistólica (mm }\end{array}$ & $\begin{array}{c}160,00 \pm \\
\mathrm{Hg})\end{array}$ & $\begin{array}{c}100,00 \pm \\
22,00\end{array}$ & $529,50 / 0,00$ \\
Presión arterial & 10,00 & & \\
diastólica (mm & $100,00 \pm$ & $65,00 \pm$ & $194,50 / 0,00$ \\
$\mathrm{Hg})$ & 10,00 & 10,00 & \\
\hline
\end{tabular}

$* \mathrm{Md} \pm$ RIC: Mediana \pm Rango Intercuartil

**Prueba no paramétrica de Mann-Whitney 
Tabla 6. Asociación entre el periodo intergenésico y la presencia de preeclampsia

\begin{tabular}{lcc}
\hline Periodo intergenésico & $\begin{array}{c}\text { Casos } \\
\mathrm{n}(\%)\end{array}$ & $\begin{array}{c}\text { Controles } \\
\mathrm{n}(\%)\end{array}$ \\
\hline Corto ( $\leq 24$ meses) & $41(25,6)$ & $26(16,1)$ \\
Adecuado (25 a 48 meses) & $33(20,6)$ & $84(52,2)$ \\
Prolongado ( $\geq 49$ meses) & $86(53,8)$ & $51(31,7)$
\end{tabular}

$\chi^{2}$ de Pearson=34,53; 2 grados de libertad; $p=0,00$

PE (Tabla 7: $\left.\chi^{2}=33,39 ; \mathrm{p}=0,00\right)$. Tener un periodo intergenésico inadecuado constituyó un factor riesgo 4,26 veces mayor de tener la PE en comparación con las gestantes con periodo intergenésico adecuado, siendo el hallazgo estadísticamente significativo $(\mathrm{OR}=$ 4,26; IC $95 \%$ : 2,59-6,98).

Tabla 7. Asociación entre el periodo intergenésico adecuado o inadecuado y la presencia de preeclampsia.

\begin{tabular}{lcc}
\hline Periodo intergenésico & $\begin{array}{c}\text { Casos } \\
\mathrm{n}(\%)\end{array}$ & $\begin{array}{c}\text { Controles } \\
\mathrm{n}(\%)\end{array}$ \\
\hline $\begin{array}{l}\text { Inadecuado ( } \leq 24 \text { meses o } \\
\geq \text { a 49 meses }\end{array}$ & $127(79,4)$ & $77(47,8)$ \\
Adecuado(25 a 48 meses) & $33(20,6)$ & $84(52,2)$ \\
\hline
\end{tabular}

$\chi^{2}$ con corrección de Yates $=33,39 ; 1$ grado de libertad; $\mathrm{p}=0,00$ $\mathrm{OR}=4,26$; IC $95 \%: 2,59-6,98$

\section{DISCUSIÓN}

La preeclampsia es una enfermedad exclusiva de la gestación humana, que conlleva a morbimortalidad perinatal elevada, y que se caracteriza por el aumento de la presión arterial y proteinuria durante la segunda mitad del embarazo.
A pesar de la importancia de la preeclampsia en términos de salud pública, ya que constituye la mayor causa de mortalidad materna en los países desarrollados, así como de morbilidad gestacional a corto y prolongado plazo, de muerte perinatal, parto pretérmino y retardo del crecimiento intrauterino, los mecanismos que la desencadenan no están totalmente precisados. Se acepta que es una enfermedad compleja, en la cual la manifestación individual de la enfermedad depende de la interacción entre varios genes maternos con el genotipo fetal y con factores medioambientales, lo que determina la generación de una respuesta que conduce a la aparición de hipertensión arterial, proteinuria y edema, signos evidentes del desarrollo de la preeclampsia. No obstante, aunque se ha acumulado evidencia sobre el aporte genético y medioambiental en la predisposición a desarrollar una preeclampsia, pocos estudios han intentado cuantificar y discriminar el peso que cada uno de ellos tiene, en relación con este aspecto, y menos clara es todavía la importancia que tienen los nuevos factores de riesgo para anticipar el surgimiento de esta enfermedad.

Los resultados de este estudio muestran que cuando existe un periodo intergenésico inadecuado hay un mayor riesgo de presentar preeclampsia. Este riesgo se presenta marcadamente alto ya que se encontró que las mujeres con periodo intergenésico inadecuado fueron cuatro veces más propensas a tener preeclampsia que las que tuvieron periodo intergenésico adecuado, siendo estas diferencias estadísticamente significativas $(\mathrm{OR}=4,26)$.

Estos resultados concuerdan con un estudio realizado por Centeno y cols. (15), quienes determinaron que existe mayor riesgo de presentar preeclampsia en mujeres con periodo intergenésico prolongado (mayor a 48 meses), en los casos se observó que un 69,2\% presentaron esta patología a diferencia de los controles que la presentaron un $38,5 \%$. 
De igual modo, Sedano y cols. (16) obtuvieron que existe asociación significativa entre el periodo intergenésico prolongado y el desarrollo de preeclampsia, siendo el periodo intergenésico prolongado un factor que eleva 4 veces el riesgo de padecer preeclampsia.

También en el estudio realizado por López y cols. (13), con 42 casos y 42 controles, la mayoría tenían periodos intergenésicos cortos o largos. A diferencia de estos autores, Mosquera y cols. (14), no encontraron significancia estadística en el periodo intergenésico como factor de riesgo de preeclampsia.

Entre los casos, el grupo etario más frecuente fue el de 19 a 29 años (52,5\%, 84 mujeres) y en los controles fue el de 30 a 42 años (96,9 \%, 156 embarazadas), datos que concuerdan con Centeno y cols. (15), quienes encontraron que las edades predominantes de los casos fueron de 18 a 35 años y la de los controles de 35 años. Por su parte, Mosquera y cols. (14) obtuvieron un promedio de edad similar en los casos (23,7 años) y en los controles (22,1 años). López y cols. (13) encontraron una edad promedio de 27 años y León (28) reportó entre pacientes con preeclampsia, un rango de edad de 20 a 34 años. Romero (27), en cambio, describió un promedio de edad de 18 años. Respecto al grado de instrucción, se observó una elevada frecuencia de bachilleres, datos similares a investigaciones previas $(13,15)$.

Al hablar del estado civil y procedencia, en su mayoría fueron solteras y de Valencia. Centeno y cols. (15) reportaron una alta frecuencia de unión estable (69,2 \%) y en el estudio de López y cols. (13), predominó el estado civil casada, de procedencia urbana, al igual que este estudio.

De las pacientes estudiadas, 10 tenían hipertensión arterial sistémica crónica como antecedente $(6,3 \%)$; lo que coincide con otros autores que reportan una frecuencia entre $8,3 \%$ y $12,8 \%$ (13 - 16). Por otro lado, León (28), encontró una frecuencia de $33 \%$ de pacientes con este antecedente.

Con respecto a los antecedentes familiares se obtuvo que 22 pacientes de los casos tenían hipertensión arterial $(13,8 \%)$ y $21(13,1 \%)$ diabetes mellitus tipo 2; Centeno y cols. (15), encontraron un 25,6\% de casos con el antecedente familiar de hipertensión arterial. Otros autores has reportado frecuencias más bajas de este antecedente familiar, entre 0 y $8 \%(13$, 14, 16), pero León (28), encontró que el $84 \%$ tenían antecedentes familiares de hipertensión arterial.

En cuanto a los antecedentes ginecoobstétricos, en 108 gestantes $(67,5 \%)$ su embarazo anterior culminó en cesárea, $102(63,4 \%)$ tenía dos o más parejas sexuales y solo 5 (3,1 \%) fumaban. De igual modo López y cols. (13) reportaron que 78,6 \% tenía cesárea anterior. Con respecto al número de parejas sexuales, es variable, encontrándose frecuencias entre $12 \%$ y $26 \%$ de dos o más parejas sexuales $(13,16)$. Centeno y cols. (15) describió un $59 \%$ de pacientes con una sola pareja.

Entre los casos, el 93,8 \% (150 gestantes), desarrolló preeclampsia con criterios de gravedad, cefalea y epigastralgia fueron los síntomas predominantes; $5,6 \%$ terminaron en eclampsia y $91,3 \%$ no presentaron complicaciones; datos similares reportaron otros autores $(16,28)$.

Se debe recordar que la atención prenatal es la piedra angular para la prevención de la preeclampsia y sus complicaciones; es fundamental que el médico lleve a cabo una vigilancia prenatal cuidadosa y dirigida a la detección y control de los factores de riesgo, para tratar de evitar los modificables y establecer medidas preventivas para evitar los no modificables, con el objetivo de lograr un diagnóstico y tratamiento temprano o una referencia oportuna. Es importante identificar el periodo intergenésico exacto de las pacientes desde la primera atención prenatal, con el fin 
de saber si este periodo es de riesgo para el embarazo y realizar promoción y prevención oportuna. Las mujeres que presentan factores de riesgo de preeclampsia como el periodo intergenésico inadecuado u otros factores de relevancia, deben ser vigiladas estrechamente durante las atenciones prenatales para así poder detectar de manera oportuna los signos y síntomas de esta enfermedad, previniendo las posibles complicaciones. Las pacientes deben recibir orientación en cuanto al periodo intergenésico, ya que no son convenientes los embarazos menores de 24 meses ni espaciar demasiado los embarazos, es decir mayor de 48 meses, por el riesgo que esto implica.

Se concluye que el periodo intergenésico inadecuado, corto o prolongado, fue frecuente en las pacientes con preeclampsia. Se demostró que las pacientes con periodo intergenésico inadecuado son 4,26 veces más propensas a desarrollar preeclampsia durante el embarazo.

\section{REFERENCIAS}

1. Moodley J. Maternal deaths due to hypertensive disorders in pregnancy. Best Pract Res Clin Obstet Gynaecol. 2008; 22(3):559-67. doi: 10.1016/j. bpobgyn.2007.11.004.

2. Organización Mundial de la Salud [Internet]. Ginebra: Mortalidad Materna. [actualizado 16/02/2018; consultado 03/05/18]; 2018. Disponible en: http:// www.who.int/mediacentre/factsheets/fs348/es/.

3. Nassif K. Obstetricia Fundamentos y Enfoque Práctico. Buenos Aires: Médica Panamericana; 2012.

4. Quispe C. Relación de ciertos factores asociados y el desarrollo de preeclampsia en gestantes atendidas en el IEMP durante el periodo agosto-noviembre del 2003 [Trabajo Especial de Grado]. Lima: Universidad Nacional Mayor de San Marcos; 2004 [consultado marzo 2019]. Disponible en: https://cybertesis.unmsm. edu.pe/bitstream/handle/20.500.12672/360/Quispe sc.pdf? sequence $=1$ \&isAllowed $=\mathrm{y}$

5. Domínguez L, Vigil P. El intervalo intergenésico: un factor de riesgo para complicaciones obstétricas y neonatales. Clin Invest Gin Obst. 2005; 32(3):122-126. doi: 10.1016/S0210-573X(05)73487-0

6. Catalyst consortium; Agencia de los EE.UU. para el Desarrollo Internacional (USAID) [Internet]. Washington:Elespaciamientoóptimo entrenacimientos: nuevos hallazgos de América Latina sobre la asociación entre intervalos entre nacimientos y la salud perinatal, materna y adolescente; 2002 [consultado abril 2019]. Disponible en: http://cat.convio.net/site/DocServer/ OBSISpaConde.pdf?docID $=140$

7. Duckitt K, Harrington D. Risk factors for pre-eclampsia at antenatal booking: systematic review of controlled studies. BMJ 2005; 330(7491):595. doi: 10.1136/ bmj.38380.674340.E0

8. Conde-Agudelo A, Belizán JM. Risk factors for preeclampsia in a large cohort of Latin American and Caribbean women. BJOG. 2000; 107(1):75-83. doi: 10.1111/j.1471-0528.2000.tb11582.x.

9. Botero J, Júbiz A, Henao G. Obstetricia y Ginecología. Texto integrado. 6ta ed. Bogotá: Ediciones Tinta Fresca; 2007.

10. Villanueva E, Alanís P. Factores pronósticos asociados a la progresión de la preeclampsia-eclampsia. Ginecol Obstet Mex [Internet]. 2000 [consultado marzo de 2019]; 68:312-316. Disponible en: https://biblat.unam. $\mathrm{mx} / \mathrm{es} / \mathrm{revista} /$ ginecologia-y-obstetricia-de-mexico/55

11. Valarino G, Mora A, Cabrera C, Durán I, Díaz Y, González S, et al. Eclampsia: morbilidad y mortalidad materna y perinatal. Rev Obstet Ginecol Venez [Internet]. 2009 [consultado marzo de 2019]; 69(3):152-161. Disponible en: http://www.sogvzla. org.ve/sogvzla $20186 / \mathrm{cms} / \mathrm{svcobtenerpdfrevista.}$ php?id $=0000000015 \&$ tipo $=$ normal\&fila $=4$

12. National High Blood Pressure Education Program Working Group Report on High Blood Pressure in Pregnancy. Am J Obstet Gynecol. 1990; 163(5 Pt 1):1691-1712. doi: 10.1016/0002-9378(90)90653-o.

13. López M, Manríquez M, Gálvez D, Ramírez E. Factores de riesgo asociados con preeclampsia. Rev Med Inst Mex Seguro Soc [Internet]. 2012 [consultado marzo 2019]; 50(5):471-476. Disponible en: https://www. redalyc.org/pdf/4577/457745497004.pdf

14. Mosquera T, Charry J. Factores de riesgo asociados a preeclampsia. ESE del Rosario. Campoalegre, Huila. 2011. RFS. 2013; 5(1):37-45. doi: https://doi. org/10.25054/rfs.v5i1.129

15. Centeno G, Crispín L. Periodo intergenésico prolongado como factor de riesgo para el desarrollo de preeclampsia en gestantes atendidas en el Instituto Nacional Materno Perinatal, enero - marzo del 2013 [Trabajo Especial de Grado]. Lima: Universidad 
Nacional Mayor de San Marcos; 2013 [consultado marzo 2019]. Disponible en: https://cybertesis.unmsm. edu.pe/bitstream/handle/20.500.12672/3010/Centeno_ hg.pdf? sequence $=1$

16. Sedano A, Ureta Y. Periodo intergenésico prolongado y preeclampsia en gestantes atendidas en el Hospital II Essalud Huancavelica [Trabajo Especial de Grado]. Lima: Universidad Nacional de Huancavelica; 2014 [consultado abril 2019]. Disponible en: https://1library. co/document/q5me0r3y-periodo-intergenesicoprolongado-preeclampsia-gestantes-atendidashospital-huancavelica.html

17. Morgan F, Calderón S, Martínez J, GonzálezA, Quevedo E. Factores de riesgo asociados con preeclampsia: estudio de casos y controles. Ginecol Obstet Mex [Internet]. 2010 [consultado marzo de 2019]; 78(3):153159. Disponible en: https://www.medigraphic.com/cgi$\mathrm{bin} / \mathrm{new} /$ resumen.cgi?IDARTICULO $=27193$

18. Laura R, Soto F. Primipaternidad de la pareja y preeclampsia en gestantes atendidas en el Hospital Departamental de Huancavelica. [Trabajo Especial de Grado]. Huancavelica: Universidad de Huancavelica; 2014 [consultado junio 2020]. Disponible en: http://repositorio.unh.edu.pe/bitstream/handle/ UNH/634/TP\%20-\%20UNH\%20OBST.\%200022. pdf? sequence $=1$ \&isAllowed $=\mathrm{y}$

19. Álvarez D, Pino L, Medina M, Rodríguez Y, Uribe A. Impacto clínico y epidemiológico de la preeclampsia y eclampsia en mujeres adolescentes primigestantes que ingresan al HUEM de la ciudad de Cúcuta entre julio y diciembre de 2011. INMBIOM [Internet]. 2016 [consultado junio de 2020]; 3(1):50-5. Disponible: http://revistas.unipamplona.edu.co/ojs_viceinves/ index.php/INBIOM/article/view/2243/1082

20. Sánchez M. Factores de riesgo para preeclampsiaeclampsia en mujeres atendidas en el Hospital Provincial General de Latacunga en el periodo comprendido entre enero 2008 a enero 2009 [Trabajo Especial de Grado]. Riobamba: Escuela Nacional Politécnica de Chimborazo; 2009 [consultado abril 2019]. Disponible en: http://dspace.espoch.edu.ec/ bitstream/123456789/192/1/94T00062.pdf
21. ACOG Practice Bulletin No. 202: Gestational Hypertension and Preeclampsia. Obstet Gynecol. 2019; 133(1):1. doi: 10.1097/AOG.0000000000003018.

22. Díaz M, Serrano D. Oportunidades de investigación en preeclampsia, desde la perspectiva de prevención primaria. Un artículo de reflexión. Rev Colomb Obstet Ginecol 2008; 59(3):206-215. doi: https://doi. org $/ 10.18597 /$ rcog. 405

23. Pacheco J. Preeclampsia/eclampsia: Reto para el ginecoobstetra. Acta Med Per [Internet] 2006 [consultado marzo de 2019]; 23(2):100-112. Disponible en: https://www.academia.edu/3615487/Preeclampsia_ eclampsia_Reto_para_el_ginecoobstetra

24. Sánchez SE. Epidemiología de la preeclampsia. Rev Per Ginecol Obstet [Internet] 2006 [consultado marzo de 2019]; 52(4):213-218. Disponible en: http://www. scielo.org.pe/pdf/rgo/v60n4/a07v60n4

25. Avena JL, Joerín VN, Dozdor LA, Brés SA. Preeclampsia - eclampsia. Rev Postg Vl Cat Med [Internet] 2007 [consultado marzo 2019]; 165:2025. Disponible en: https://med.unne.edu.ar/revistas/ revista165/5 165.pdf

26. Basso O, Christensen K, Olsen J. Higher risk of preeclampsia after change of partner. An effect of longer interpregnancy intervals? Epidemiology. 2001; 12(6):624-9. doi: 10.1097/00001648-20011100000008.

27. Romero M. Prevalencia de adolescentes embarazadas con diagnóstico de preeclampsia severa en la maternidad de Alto Riesgo Obstétrico Hugo Chávez en el periodo mayo-agosto 2016. [Trabajo Especial de Grado]. Valencia (Carabobo): Universidad de Carabobo; 2016.

28. León O. Incidencia de preeclampsia en pacientes con antecedentes de trastorno hipertensivo en embarazos previos controladas en la consulta de SIEMPRE de la Maternidad de Alto Riesgo de la Ciudad Hospitalaria "Dr Enrique Tejera" durante el periodo abril 2014 hasta diciembre 2017 [Trabajo Especial de Grado]. Valencia (Carabobo): Universidad de Carabobo; 2018.

Recibido 20 de septiembre de 2020 Aprobado 10 de diciembre de 2020 